\title{
Pulmonary artery dissection in a patient with Eisenmenger's syndrome and successful conservative medical treatment
}

\author{
Sun Hwa Hong ${ }^{1}$ and Kyung-Hee Kim²
}

${ }^{1}$ Department of Radiology, ${ }^{2}$ Division of Cardiology, Department of Internal Medicine, Sejong General Hospital, Bucheon, Korea

Received: August 4, 2016

Revised : August 26, 2016 Accepted: September 3, 2016

\section{Correspondence to}

Kyung-Hee Kim, M.D.

Tel: +82-32-340-1443

Fax: +82-32-340-1180

E-mail: learnbyliving9@gmail.com
A 43-year-old woman was referred to our emergency department with acute onset of dyspnea, palpitation and chest discomfort. The patient did not have any pre-existing medical history nor any complaint of a particular symptom 1 day before the hospital visit. Physical examination revealed a pulse rate of 117 beats per minute, blood pressure of $100 / 60 \mathrm{mmHg}$, and pulse oximetry reading of $88 \%$. A diagnosis of right ventricular hypertrophy and dilatation of the right ventricle (RV) along with severe RV deterioration was made on transthoracic echocardiography. Severe pulmonary hypertension was diagnosed with pulmonary artery $(\mathrm{PA})$ pressure of approximately $90 \mathrm{mmHg}$ by tricuspid regurgitation. An enlargement of the $\mathrm{PA}$, an extensive intimal flap inside the proximal portion of the right lower lobe interlobar PA, and peripheral involvement of the superior and medial basal segmental arteries were revealed after an urgent multidetector computed tomography (MDCT) pulmonary angiography under the suspicion of pulmonary embolism (Fig. 1). Chronic thromboembolism within the right middle lobar PA and its segmental branches was accu-
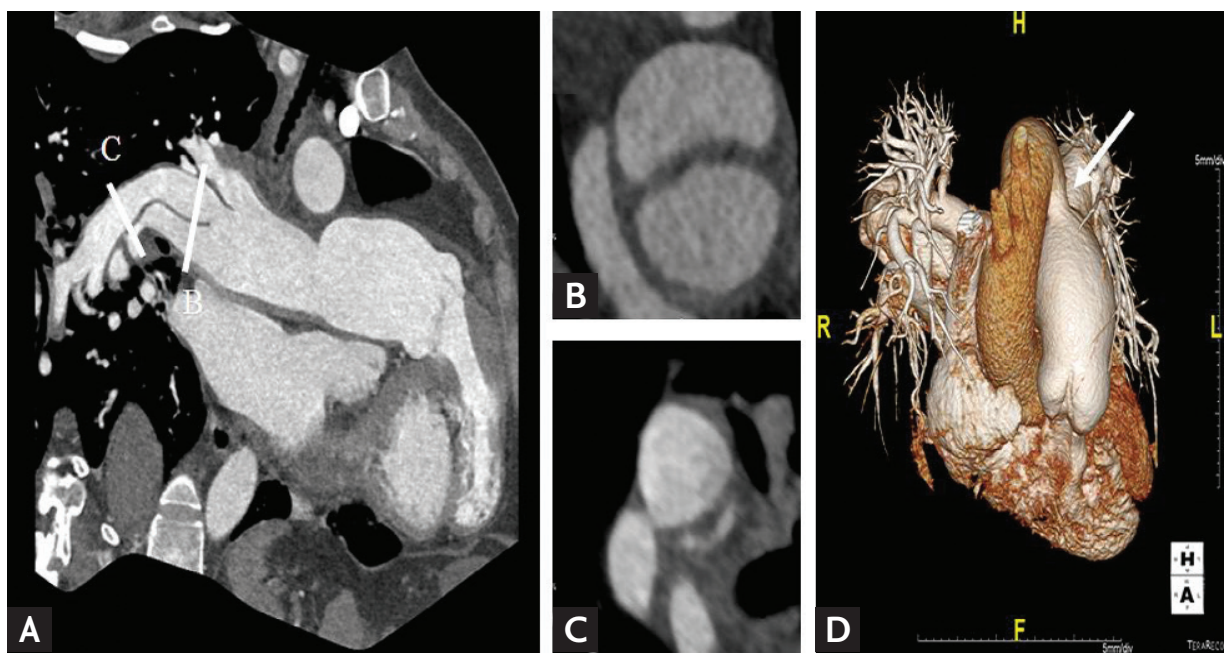

Figure 1. (A) Multidetector computed tomography pulmonary angiography shows a extensively dissected right pulmonary artery with the presence of an intimal flap, (B) starting from the right lower lobe artery, (C) the superior segmental artery and the medial basal segmental artery. (D) A three dimensional volume rendering image demonstrates a large PDA (arrow) and dilated tortuous pulmonary arteries. 


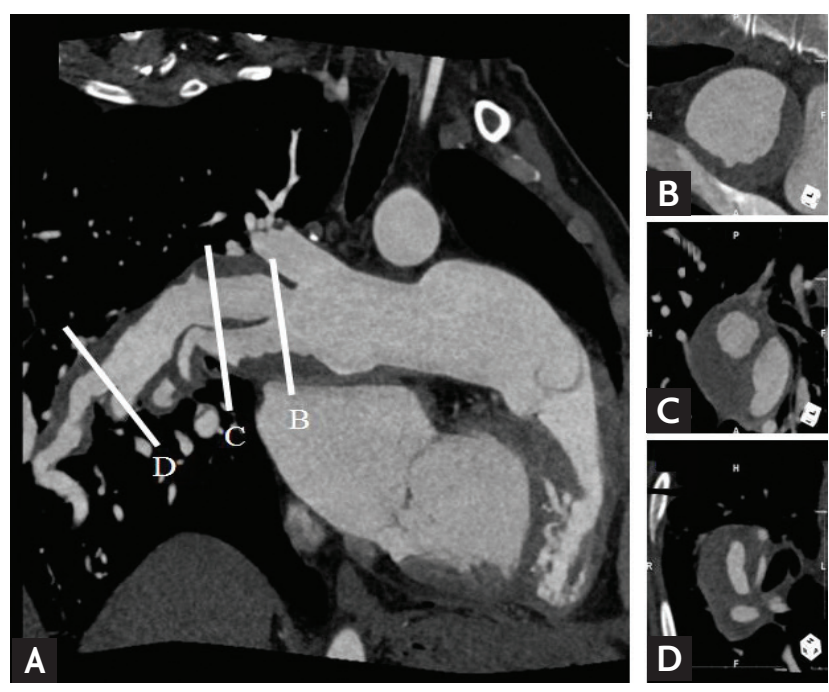

Figure 2. (A-D) Follow-up multidetector computed tomography pulmonary angiography shows progression of partial thrombosis in the false lumen. rately displayed on MDCT along with a large PDA. The patient was treated with oxygen and endothelin receptor antagonist, phosphodiesterase 5 inhibitors, and antibiotics for combined inflammation. A follow-up MDCT and cardiac magnetic resonance imaging showed progression of pulmonary hemorrhage and thrombosis in the PA up to its intersegmental branches (Fig. 2, Supplementary Video 1). An adjustment of medicine was priorly considered before the need for heart-lung transplantation. Fortunately, the patient was discharged in a clinically stable condition 1 month after proper medical treatment. After a 2-year follow-up period, she remained stable without any complications and blood examination was normalized including C-reactive protein.

\section{Conflict of interest}

No potential conflict of interest relevant to this article was reported. 
Hong SH and Kim KH. Conservative treatment of PA dissection

Supplementary Video 1. Magnetic resonance imaging shows dissection of the pulmonary artery with an intimal flap. 